PROCEEDINGS OF THE

AMERICAN MATHEMATICAL SOCIETY

Volume 131, Number 7, Pages 2279-2287

S 0002-9939(02)06751-5

Article electronically published on December 30, 2002

\title{
NORMAL SUBSPACES OF PRODUCTS OF FINITELY MANY ORDINALS
}

\author{
WILLIAM G. FLEISSNER
}

(Communicated by Alan Dow)

\begin{abstract}
Let $X$ be a subspace of the product of finitely many ordinals. If $X$ is normal, then $X$ is strongly zero-dimensional, collectionwise normal, and shrinking. The proof uses $\left(\kappa_{1}, \ldots, \kappa_{n}\right)$-stationary sets.
\end{abstract}

\section{Preliminary}

We use $\left(\kappa_{1}, \ldots, \kappa_{n}\right)$-stationary sets to prove the following theorem which extends results of Kemoto, Nogura, Smith, and Yajima in [4] and Stanley in [8].

Theorem 1.1. Let $X$ be a subspace of the product of finitely many ordinals. The following are equivalent:

(1) $X$ is normal.

(2) $X$ is normal and strongly zero-dimensional.

(3) $X$ is collectionwise normal.

(4) $X$ is shrinking.

This theorem differs from the theorem of [4] in two ways. First, that theorem applies to subspaces of the product of two ordinals. Second, that theorem does not include "strongly zero-dimensional". Instead, the fourth condition asserts that nine types of pairs of closed sets are separated. For example, if $(\mu, \nu) \notin X \subseteq \lambda^{2}$, then $\{(\alpha, \nu) \in X: \alpha<\lambda\}$ and $\{(\mu, \beta) \in X: \beta<\lambda\}$ are separated. Stanley's theorem asserts that if $X$ is a normal subspace of the product of finitely many ordinals, then $X$ is collectionwise Hausdorff. The condition that $X$ is strongly zero-dimensional cannot be added to Theorem 1.1 because the paper [3] describes a subspace of $\omega+1 \times \mathfrak{c}$ which is not strongly zero-dimensional.

First we define the notions in Theorem 1.1, and then introduce notation for tuples and products.

Definition 1.2. A shrinking of a cover $\mathcal{A}$ of a space $X$ is a cover $\mathcal{B}=\left\{B_{A}: A \in \mathcal{A}\right\}$ such that $c l B_{A} \subseteq A$ for all $A \in \mathcal{A}$. Every finite cozero cover of a space $X$ has a cozero shrinking. If $X$ is normal, then every finite open cover of $X$ has an open shrinking (see [1], p. 386). We say that a space $X$ is shrinking if every open cover

Received by the editors May 11, 2000 and, in revised form, February 22, 2002.

2000 Mathematics Subject Classification. Primary 54B10, 54D15, 03 E10.

Key words and phrases. Normal, collectionwise normal, shrinking, stationary set, pressing down lemma, finite product of ordinals. 
of $X$ has an open shrinking. We say that a space $X$ is normal and strongly zerodimensional if whenever $H_{0}$ and $H_{1}$ are disjoint closed subsets of $X$, then there are disjoint clopen sets $W_{0}$ and $W_{1}$ such that $H_{0} \subseteq W_{0}, H_{1} \subseteq W_{1}$, and $W_{0} \cup W_{1}=X$. The following characterization will be useful: a space $X$ is normal and strongly zerodimensional iff every finite open cover of $X$ has a clopen shrinking. We say that a space $X$ is collectionwise normal if whenever $\mathcal{H}$ is a discrete collection of closed subsets of $X$, then there is a discrete collection of open sets $\mathcal{U}=\{U(H): H \in \mathcal{H}\}$ such that $H \subseteq U(H)$ for all $H \in \mathcal{H}$.

Definition 1.3. Let $a$ be an $n$-tuple of ordinals. Let $\Pi a$ abbreviate $\prod_{i \leq n} a_{i}$ and let $\Pi a^{*}$ abbreviate $\prod_{i \leq n}\left(\{-1\} \cup a_{i}\right)$. If $y \in \Pi a$ and $z \in \Pi a^{*}$ we define

$$
\begin{gathered}
z<y \quad \text { iff } \quad z_{i}<y_{i} \text { for all } i \leq n, \\
z \leq y \quad \text { iff } \quad z_{i} \leq y_{i} \text { for all } i \leq n, \\
(z, y] \quad=\quad\{t \in \Pi a: z<t \leq y\}, \\
b \prec a \quad \text { iff } \quad b \leq a \text { and } b_{i}<a_{i} \text { for some } i .
\end{gathered}
$$

"Intervals" $(z, y]$ form a basis for the product topology on $\Pi a$. We will prove Theorem 1.1 by induction on the well founded order $\prec$.

We set notation for concatenating $n$-tuples. If $a=\left(a_{1}, \ldots, a_{m}\right)$ and $b=$ $\left(b_{1}, \ldots, b_{n}\right)$, then $a \frown b$ is the $(m+n)$-tuple $c=\left(c_{1}, \ldots, c_{m+n}\right)$, where $c_{i}=a_{i}$ for $i \leq m$ and $c_{m+j}=b_{j}$ for $j \leq n$. If $X \subseteq \Pi(a-b)$ and $t \in \Pi a$, define $X_{t}=\{y \in \Pi b: t \frown y \in X\}$.

In Section 2, we introduce the notion $k$-stationary, where $k$ is a strictly increasing $n$-tuple of regular uncountable cardinals. In section 3 , we describe a class of nonnormal spaces. The fact that these spaces are not normal leads to a dicotomy for normal subspaces $X$ of $\Pi a$ : either they are "reducible" to a free sum of spaces which are homeomorphic to subspaces of $\Pi b$ with $b \prec a$ (Lemma 3.7), or we can apply a general Pressing Down Lemma (Lemma [2.2, Lemma [3.10). Preparations complete, we prove Theorem 1.1. The implication [(3) or (4)] $\rightarrow(1)$ is obvious; we prove $(1) \rightarrow(2)$ in Section 4; and we prove $(2) \rightarrow[(3)$ and (4)] in Section 5.

\section{Stationary}

The original proof of Theorem 1.1 used a more general theory of $\left(\kappa_{1}, \ldots, \kappa_{n}\right)$ stationary sets, developed in Section 3 of [2]. Later, we realized that Lemma 3.5] allows us to specialize to the case where the $\kappa_{i}$ 's are strictly increasing, where the theory is simpler. We prove only what is needed for Theorem 1.1, using a different definition from that of [2]. The theory of $\left(\kappa_{1}, \ldots, \kappa_{n}\right)$-stationary sets, for non-decreasing $n$-tuples of regular, uncountable cardinals, is presented in [3], where we show that $A_{1} \times A_{2} \times \ldots \times A_{n}$ is strongly zero-dimensional when each $A_{i}$ is a subspace of an ordinal.

Definition 2.1. For $X \subseteq \Pi b \times \kappa$, we define the stationary projection

$$
\text { st } \pi[X]=\left\{t \in \Pi b: X_{t} \text { is stationary in } \kappa\right\} .
$$

Let $k=\left(\kappa_{1}, \ldots, \kappa_{n}\right)$ be a strictly increasing $n$-tuple of regular uncountable cardinals. We define $Y$ to be $k$-stationary by induction on $n$. For $Y \subseteq \Pi\left(\kappa_{1}\right)=\kappa_{1}$, we say that $Y$ is $k$-stationary iff $Y$ is stationary in $\kappa_{1}$. For $Y \subseteq \Pi k \times \kappa$, we say that $Y$ is $k^{\frown} \kappa$-stationary iff st $\pi[Y]$ is $k$-stationary. Sometimes it is convenient to say that $Y=\{0\}$ is 0 -stationary, where 0 is the empty sequence. 
Lemma 2.2. Let $Y \subseteq \Pi k$ be $k$-stationary, where $k=\left(\kappa_{1}, \ldots, \kappa_{n}\right)$ is a strictly increasing $n$-tuple of regular uncountable cardinals. Then:

(1) (Kemoto) If $f: Y \rightarrow \Pi k^{*}$ satisfies $f(y)<y$ for all $y \in Y$, then there are $q \in \Pi k$ and $Y^{\prime}$, a k-stationary subset of $Y$ such that $f(y)<q \leq y$ for all $y \in Y^{\prime}$.

(2) If $\theta<\kappa_{1}$ and $g: Y \rightarrow \theta$, then there are $\beta \in \theta$ and $Y^{\prime}$, a $k$-stationary subset of $Y$ such that $g(y)=\beta$ for all $y \in Y^{\prime}$.

(3) $Y$ is a directed, cofinal subset of $\Pi k$.

(4) $S=\{y \in Y: \Pi y \cap Y$ is not cofinal in $\Pi y\}$ is not $k$-stationary.

Proof. By induction on $n$. If $n=1$, this is the usual Pressing Down Lemma (PDL) (see [7, II, 6.15). Let $Y$ be $k^{\frown} \kappa$-stationary. For each $t \in \operatorname{st} \pi[Y]$, use PDL to find $s_{t}<t, \xi_{t}<\kappa$, and $Z_{t}$, a $\kappa$-stationary subset of $Y_{t}$ such that $f(t \smile \xi)=s_{t} \xi_{t}$ for all $\xi \in Z_{t}$. By induction hypothesis, there are $r \in \Pi k$ and $T^{\prime}$, a $k$-stationary subset of $T$ such that $s_{t}<r \leq t$ for all $t \in T^{\prime}$. Let $\xi=\sup \left\{\xi_{t}: t \in T^{\prime}\right\}$. Set $q=r \frown \xi$.

The similar proof of clause 2 is left to the reader. Clause 3 is obvious.

Towards a contradiction, assume that $S$ is $k$-stationary. For each $y \in S$, let $f(y) \in \Pi y$ satisfy $[f(y), y) \cap Y=\emptyset$. Apply clause 1 to get $q$ and a stationary subset $Y^{\prime}$ of $S$. Apply clause 3 twice to find $y$ and $y^{\prime}$ in $Y^{\prime}$ such that $q<y^{\prime}<y$. Then $y^{\prime} \in[f(y), y) \cap Y$. Contradiction!

\section{NORMAL}

Lemma 3.1. Let $X \subseteq \Pi b \times \kappa$, where $|\Pi b|<\kappa$ and $\kappa$ is a regular, uncountable cardinal. If $X$ is normal, then $T=\operatorname{st} \pi[X]$ is normal.

Proof. For each $s \in \Pi b \backslash T$, choose $C_{s}$ club in $\kappa$ such that $\{s\} \times C_{s} \cap X=\emptyset$. Set $C=\bigcap\left\{C_{s}: s \in \Pi b \backslash T\right\}$. Let $H_{0}$ and $H_{1}$ be disjoint closed subsets of $T$. Set $\tilde{H}_{0}=\left(H_{0} \times C\right) \cap X$ and $\tilde{H}_{1}=\left(H_{1} \times C\right) \cap X$. Let $\tilde{U}_{0}$ and $\tilde{U}_{1}$ be disjoint, open in $X$ with $\tilde{H}_{0} \subseteq \tilde{U}_{0}$ and $\tilde{H}_{1} \subseteq \tilde{U}_{1}$. We will show that $U_{0}=\operatorname{st} \pi\left[\tilde{U}_{0}\right]$ and $U_{1}=\operatorname{st} \pi\left[\tilde{U}_{1}\right]$ are disjoint open subsets of $T$.

Let $t \in U_{e}$. For each $\gamma$ such that $t^{-} \gamma \in \tilde{U}_{e}$, find $s_{t, \gamma}<t$ and $\beta_{t, \gamma}<\gamma$ satisfying $X \cap\left(\left(s_{t, \gamma}, t\right] \times\left(\beta_{t, \gamma}, \gamma\right]\right) \subseteq \tilde{U}_{e}$. By PDL, there are $Y_{t}$, stationary in $\kappa, s_{t}<t$ and $\beta_{t(e)} \in \kappa$ so that $s_{t, \gamma}=s_{t}$ and $\beta_{t, \gamma}=\beta_{t(e)}$ for all $\gamma \in Y_{t}$. Then $\left(s_{t}, t\right] \cap T \subseteq U_{e}$, so $U_{e}$ is open. If $t \in U_{0} \cap U_{1}$, then $\gamma \in \tilde{U}_{0} \cap \tilde{U}_{1}$ for all $\gamma>\max \left\{\beta_{t}(0), \beta_{t}(1)\right\}$. Contradiction!

Definition 3.2. For sets $S$ and $T$, let the diagonal map $\operatorname{Dg}_{T}: S \rightarrow S^{T}$ be defined by $\operatorname{Dg}_{T}(s)(t)=s$ for all $t \in T$. For $X \subseteq S^{T}$, set $\Delta_{T}(X)=\left\{s \in S: \operatorname{Dg}_{T}(s) \in X\right\}$. We will omit subscripts when it is clear from the context. Let Lim be the set of countable limit ordinals. For $A$ a set of ordinals, let $L(A)$ be the set of ordinals $\xi$ (not necessarily in $A$ ) such that every neighborhood of $\xi$ meets $A$ in an infinite set.

Example 3.3. Let $X=\left\{(\mu, \nu) \in \omega_{1} \times \omega_{1}: \mu<\nu\right\}$. Let $H_{0}=\{(\mu, \mu+1) \in X$ : $\mu \in \operatorname{Lim}\}$ and $H_{1}=\{(\mu, \nu) \in X: \nu \in \operatorname{Lim}\}$.

This space $X$ is a specific instance of a class of nonnormal spaces (implicit in Lemma 4 of [4], and Lemma 5.1.3 of [5]). Let $\kappa$ be an uncountable regular cardinal. Let $S \subseteq C \subseteq \kappa$, where $S$ is stationary and $C$ is club. Suppose that $X \subseteq\left\{(\mu, \nu) \in \kappa^{2}: \mu \leq \nu\right\} \backslash C^{2}$ and that $X_{\mu}$ is stationary for every $\mu \in S$. Finally, suppose that $h(\mu) \in X_{\mu} \cap \bigcap_{\mu^{\prime}<\mu} L\left(X_{\mu^{\prime}}\right)$ satisfies $(\mu, h(\mu)] \cap C=\emptyset$ for each 
$\mu \in S$. We claim that $X$ is not normal because $H_{0}=\{(\mu, h(\mu)): \mu \in S\}$ and $H_{1}=\{(\mu, \nu) \in X: \nu \in C\}$ cannot be separated in $X$.

Towards proving the claim, let $H_{0} \subseteq U_{0}$ open. For each $\mu \in S$, find $f(\mu)<\mu$ and $\mu \leq g(\mu)<h(\mu)$ so that $(f(\mu), \mu] \times(g(\mu), h(\mu)] \subseteq U_{0}$. Apply PDL to $f$ and $S$ to get $S^{\prime}$ and $\beta$ so that $f(\mu)=\beta$ for all $\mu \in S^{\prime}$. Fix $\mu_{0} \in S \backslash(\beta+1)$. Let $\nu_{0} \in L\left(S^{\prime}\right) \cap X_{\mu_{0}}$. Then $\left(\mu_{0}, \nu_{0}\right) \in H_{1}$. We claim that $\left(\mu_{0}, \nu_{0}\right) \in \operatorname{cl} U_{0}$.

For every neighborhood $N$ of $\left(\mu_{0}, \nu_{0}\right)$, there is $\gamma \in\left[\mu_{0}, \nu_{0}\right)$ such that $\left\{\mu_{0}\right\} \times$ $\left(\gamma, \nu_{0}\right] \subseteq N$. Choose $\mu_{1} \in S^{\prime} \cap\left(\gamma, \nu_{0}\right)$. Because $h\left(\mu_{1}\right) \in L\left(X_{\mu_{0}}\right)$, there is $\nu_{1} \in$ $\left(g\left(\mu_{1}\right), h\left(\mu_{1}\right)\right) \cap X_{\mu_{0}}$. Then $\left(\mu_{0}, \nu_{1}\right) \in N \cap U_{0}$.

Lemma 3.4. Let $X$ be a normal subspace of $\kappa^{2}$, where $\kappa$ is an uncountable regular cardinal. If $\Delta(X)$ is not stationary, then there is $C$, club in $\kappa$, such that $X \cap C^{2}=\emptyset$.

Proof. Let $C^{\prime}$ be club so that $\operatorname{Dg}\left[C^{\prime}\right] \cap X=\emptyset$. Set $X^{\prime}=\{(\mu, \nu) \in X: \mu \leq \nu\}$. Towards a contradiction, assume that $S^{\prime}=\left\{\mu \in \kappa: X_{\mu}\right.$ is stationary $\}$ is stationary. (In the terminology of 2], assume that $X^{\prime}$ is $\kappa^{2}$-stationary.) For each $\mu \in S^{\prime}$, choose $h(\mu) \in X_{\mu}^{\prime} \cap \bigcap_{\mu^{\prime}<\mu} L\left(X_{\mu^{\prime}}\right)$. Set $C=\left\{\gamma \in C^{\prime}:(\forall \mu<\gamma)(h(\mu)<\gamma)\right\}$. Then $S^{\prime} \cap C$, $C, X^{\prime}$, and $h$ satisfy the conditions of Example [3.3. Hence $X$ is not normal. Contradiction!

Therefore there are clubs $C^{*}$ and $\left(C_{\mu}\right)_{\mu \in C^{*}}$ such that $C_{\mu} \cap X_{\mu}=\emptyset$ for all $\mu \in C^{*}$. Set $E_{1}=\left\{\gamma \in C^{*}:(\forall \mu<\gamma)\left(\gamma \in C_{\mu}\right)\right\}$. Then $E_{1}$ is club and $E_{1}^{2} \cap\left(X^{\prime}\right)^{2}=\emptyset$.

By a similar argument, we get a club $E_{2}$ so that $E_{2}^{2} \cap\{(\mu, \nu) \in X: \mu \geq \nu\}=\emptyset$. Then $C^{\prime} \cap E_{1} \cap E_{2}$ is the desired club.

Lemma 3.5. Let $X$ be a normal subspace of $\kappa^{n}$, where $\kappa$ is an uncountable regular cardinal and $n \in \omega$. If $\Delta(X)$ is not stationary, then there is $C$, club in $\kappa$, such that $X \cap C^{n}=\emptyset$.

Proof. By induction on $n$. The cases $n=0$ and $n=1$ are trivial; the case $n=2$ is Lemma 3.4 Let $X$ be a normal subspace of $\kappa^{n}$ where $n=m+1$. For each $e \in m^{n}$, let $X_{e}=\left\{x \in X\right.$ : if $e(i)=e(j)$, then $\left.x_{i+1}=x_{j+1}\right\}$. Each $X_{e}$ is a closed, hence normal, subspace of $X$ and is homeomorphic to a subspace of $\kappa^{m}$, so by induction hypothesis, there is a club $C_{e}$ such that $C_{e}^{m} \cap X_{e}=\emptyset$. Set $G=\bigcap\left\{C_{e}: e \in m^{n}\right\}$.

Fix $\mu \in G$ and $i \leq n$. Set $X(\mu, i)=\left\{x \in X: x_{i}=\mu\right\}$, a closed, normal subspace of $X$. Let $h$ be the natural homeomorphism of $X(\mu, i)$ into $\kappa^{m}$ (- $h$ "deletes $\mu$ in the $i^{\text {th }}$ place"). It is straightforward to verify that $\operatorname{Dg}_{m}[G] \cap h[X(\mu, i)]=\emptyset$. (Consider the $e \in 2^{n}$ satisfying $e(i)=0$ and $e(j)=1$ if $j \neq i$.) By induction hypothesis there is a club $C(\mu, i)$ such that if $z_{j} \in C(\mu, i)$ for all $j \neq i$, then $z \notin X(\mu, i)$. Unfix $\mu$ and $i$. Then $C=\{\gamma \in G:(\forall \mu \in \gamma \cap G)(\forall i \leq n)(\gamma \in C(\mu, i))\}$ is the desired club.

Now we introduce the notion "reducible" and prove a lemma justifying the name.

Definition 3.6. If $\alpha=\beta+1$, we say that $\{\beta\}$ is club in $\alpha$ and that $\operatorname{cof} \alpha=1$. Let $X \subseteq \Pi a$, where $a$ is an $n$-tuple of ordinals. We say $X$ is reducible in $a$, and we write $\operatorname{red}_{a}(X)$, when either cof $a_{i}=\omega$ for some $i \leq n$, or there are $C_{i}, i \leq n$, each $C_{i}$ club in $a_{i}$ such that $C_{1} \times \ldots \times C_{n} \cap X=\emptyset$.

Lemma 3.7. If $X$ is a normal and strongly zero-dimensional subspace of $\Pi$, and $\operatorname{red}_{a}(X)$, then $X$ is homeomorphic to a free sum $\bigoplus\left\{Y_{\lambda}: \lambda \in \Lambda\right\}$ where $Y_{\lambda} \subseteq \Pi b_{\lambda}$ and $b_{\lambda} \prec a$ for each $\lambda$.

Proof. It is clear if cof $a_{i}=\omega$ for some $i$. 
Otherwise, let $C_{i}, i \leq n$, be clubs witnessing $\operatorname{red}_{a}(X)$. For each $i$, set $Z_{i}=\{z \in$ $\left.\Pi a: z_{i} \notin C_{i}\right\}$. Because $X$ is normal and strongly zero-dimensional, the finite open cover $\left\{Z_{i} \cap X: i \leq n\right\}$ has a disjoint clopen refinement $\left\{W_{i}: i \leq n\right\}$. For each $i \leq n, W_{i} \subseteq Z_{i}$ is homeomorphic to a subspace of the free sum of spaces $\Pi b$ with $b \prec a$.

Let $X \subseteq \Pi a$, where $a$ is an $n$-tuple of ordinals. If cof $a_{i}=\omega$ for some $i \leq n$, then $\operatorname{red}_{a}(X)$. If $a=\left(\beta_{1}+1, \ldots, \beta_{n}+1\right)$, then $\operatorname{red}_{a}(X)$ iff $\left(\beta_{1}, \ldots, \beta_{n}\right) \notin X$. There remains the case where $\operatorname{cof} a_{i} \neq \omega$ for all $i \leq n$ and $\operatorname{cof} a_{i}>\omega$ for some $i \leq n$. Now our goal is to show that in this case, it suffices to consider nondecreasing $m$-tuples of regular, uncountable cardinals.

Let us say that $\left(d, \rho,\left(\mu_{j}: j \leq m\right), \psi\right)$ is good for $a$ if the following are satisfied:

(1) $d$ is a nondecreasing $m$-tuple of regular, uncountable cardinals,

(2) $\rho:\{1, \ldots, m\} \rightarrow\{1, \ldots, n\}$ is one to one,

(3) for all $j \leq m, \mu_{j}: d_{j} \rightarrow a_{\rho(j)}$ is increasing, continuous, and cofinal,

(4) for all $i \leq n$, if $i \notin \operatorname{ran} \rho$, then $a_{i}=\beta_{i}+1$,

(5) $\psi: \Pi d \rightarrow \Pi a$ satisfies for all $y \in \Pi d,(\psi(y))_{i}=\mu_{j}\left(y_{j}\right)$ if $\rho(j)=i ;(\psi(y))_{i}=$ $\beta_{i}$ otherwise.

Lemma 3.8. Assume that $\left(d, \rho,\left(\mu_{j}: j \leq m\right), \psi\right)$ is good for a. Let $d=\left(\kappa_{1}, \ldots, \kappa_{m}\right)$. If $X$ is a normal subspace of $\Pi$ a, then $X \cap \operatorname{ran} \psi$ is closed in $X$, hence normal, and then $\psi^{\leftarrow}[X]$ is normal. If $C_{1} \times \ldots \times C_{m}$, each $C_{i}$ club in $\kappa_{i}$ witnesses $\operatorname{red}_{d}\left(\psi^{\leftarrow}[X]\right)$, then $\psi\left[C_{1} \times \ldots \times C_{m}\right]$ witnesses red red $_{a}(X)$.

Lemma 3.9. Let a be an n-tuple of ordinals. If $X$ is a normal subspace of $\Pi a$, and not red $\operatorname{rad}_{a}(X)$, then there are $Y$ and $\varphi$ such that $Y$ is $a\left(\kappa_{1}, \ldots, \kappa_{p}\right)$-stationary set and $\varphi$ is an order preserving homeomorphism from $Y$ into $X$ such that $\varphi[Y]$ is closed and cofinal in $X$.

Proof. If $a=\left(\beta_{1}+1, \ldots, \beta_{n}+1\right)$, let $Y=\{0\}$. Otherwise, by Lemma 3.8 we may assume that $a$ is a nondecreasing $n$-tuple of regular cardinals. Let $\left(\kappa_{1}, \ldots, \kappa_{p}\right)$ be the distinct values of $a_{i}$ in strictly increasing order. We proceed by induction on $p$. When $p=1$, then $\Pi a=\kappa_{1}^{n_{1}}$, and Lemma 3.5 says that $Y=\Delta(X)$ is stationary in $\kappa_{1}$. Let $\varphi=\mathrm{Dg}$; then $\varphi[Y]$ is closed in $X$.

When $p=p^{\prime}+1$, then $a=b \frown c$, where $c_{i}=\kappa_{p}>|\Pi b|$ for all $i \leq n_{p}$. Consider $T=$ $\left\{t \in \Pi b: \Delta\left(X_{t}\right)\right.$ is stationary in $\left.\kappa_{p}\right\}$. If $\operatorname{red}_{b}(T)$, then $\operatorname{red}_{a}(X)$. Contradiction! So we may apply the induction hypothesis to $T$ to obtain a $\left(\kappa_{1}, \ldots, \kappa_{p^{\prime}}\right)$-stationary set $T^{\prime}$, closed in $\Pi b$, and a homeomorphism $\varphi^{\prime}$. Set $Y=\left\{z \frown \xi \in T^{\prime} \times \kappa_{p}: \operatorname{Dg}(\xi) \in\right.$ $\left.X_{z}\right\}=\left(T^{\prime} \times \operatorname{Dg}\left[\kappa_{p}\right]\right) \cap X$. Define $\varphi$ by $\varphi(z \frown \xi)=\varphi^{\prime}(z) \frown \operatorname{Dg}(\xi)$.

Lemma 3.10. Let $X, Y$ and $\varphi$ satisfy the conclusion of Lemma 3.9 Let $f$ : $\varphi[Y] \rightarrow \Pi a^{*}$ satisfy $f(\varphi(y))<\varphi(y)$ for all $y \in Y$. Then there are $q \in \Pi a$ and $R$, directed and cofinal in $X$, such that $f(x)<q \leq x$ for all $x \in R$.

Proof. It is easy to verify the conclusions when $Y=\{0\}$. Otherwise, let $Y_{0}=\{y \in$ $Y: \Pi y \cap Y$ is cofinal in $\Pi y\}$. By Lemma $2.2 Y_{0}$ is $k$-stationary. For each $y \in Y_{0}$, find $g(y) \in Y$ such that $f(\varphi(y)) \leq \varphi(g(y))<\varphi(y)$. Apply Lemma 2.2(1) to $Y_{0}$ and $g$ to get $q^{\prime}$ and $Y^{\prime}$. Set $q=\varphi\left(q^{\prime}\right)$ and $R=\varphi\left[Y^{\prime}\right]$.

Of course, if $X$ satisfies the hypothesis of Lemma 3.9 and $f$ is defined on all of $X$, we may apply this Lemma without explicitly naming $Y$ and $\varphi$. 


\section{StRONGLY ZERO-DIMENSIONAL}

In this section we prove that (1) implies (2) of Theorem 1.1 following the method of [2]. Let $H(Z)$ abbreviate "For all $X \subseteq Z$, if $X$ is normal, then $X$ is strongly zero-dimensional". The next lemma lists some methods to prove $H(Z)$ for "big" spaces from $H\left(Z^{\prime}\right)$ for "small" spaces.

Lemma 4.1. Each of the following are sufficient to imply $H(Z)$ :

(1) $Z$ is homeomorphic to a subset of $Z^{\prime}$ and $H\left(Z^{\prime}\right)$.

(2) $Z=\bigoplus_{i \in I} Z_{i}$, and $H\left(Z_{i}\right)$ for all $i \in I$.

(3) $Z=Z_{1} \cup Z_{2}, H\left(Z_{1}\right), H\left(Z_{2}\right)$, and $Z_{1}$ is closed in $Z$.

(4) $Z=\bigcup_{i \in I} Z_{k}$, I is finite, and $Z_{i}$ is open in $Z$ for all $i \in I$.

Proof. Clauses 1 and 2 are obvious. Clause 4 follows from clause 3 .

Towards clause 3 , let $H_{0}$ and $H_{1}$ be disjoint closed subsets of a normal subspace $X$ of $Z$. Then $X_{1}=X \cap Z_{1}$ is normal, so by $H\left(Z_{1}\right)$ there are disjoint closed $K_{0}$ and $K_{1}$ satisfying

$$
H_{0} \cap X_{1} \subseteq K_{0}, \quad H_{1} \cap X_{1} \subseteq K_{1}, \quad \text { and } K_{0} \cup K_{1}=X_{1} .
$$

Since $X$ is normal, there are open $U_{0}$ and $U_{1}$ such that $H_{0} \cup K_{0} \subseteq U_{0}, H_{1} \cup K_{1} \subseteq U_{1}$, and $\operatorname{cl} U_{0} \cap \operatorname{cl} U_{1}=\emptyset$. Set $X_{2}=X \backslash\left(U_{0} \cup U_{1}\right)$, a closed, hence normal, subspace of $X$. By $H\left(Z_{2}\right)$, there are disjoint $W_{0}$ and $W_{1}$, clopen in $X_{2}$, satisfying

$$
\left(\operatorname{cl} U_{0} \cup H_{0}\right) \cap X_{2} \subseteq W_{0}, \quad\left(\operatorname{cl} U_{1} \cup H_{1}\right) \cap X_{2} \subseteq W_{1}, \quad \text { and } W_{0} \cup W_{1}=X_{2} .
$$

Then $U_{0} \cup W_{0}$ and $U_{1} \cup W_{1}$ are the desired clopen subsets of $X$.

Because every product of finitely many ordinals is a subspace of $\alpha^{n}$ for some $n$ and $\alpha$, to prove (1) implies (2) of Theorem 1.1 it suffices to prove $(\forall n \in \omega) H\left(\alpha^{n}\right)$ for all ordinals $\alpha$. We proceed by induction. The base step is easy: if $\alpha$ is countable, then every subspace of $\alpha^{n}$ is strongly zero-dimensional. For the induction steps, we use Lemma 4.3 when $\alpha$ is an uncountable regular cardinal, and Lemma 4.2 otherwise.

Lemma 4.2. Let $\alpha$ be either a successor ordinal or a singular limit ordinal. If $(\forall m \in \omega) H\left(\beta^{m}\right)$ for all $\beta<\alpha$, then $(\forall n \in \omega) H\left(\alpha^{n}\right)$.

Proof. We prove $(\forall m \in \omega) H\left(\beta^{m} \times \alpha^{n}\right)$ by induction on $n$. The base step $n=0$ follows from hypothesis. Let $n=p+1$.

If $\alpha$ is a successor, $\alpha=\gamma+1$, say, then set $C=\{\gamma\}$. If $\alpha$ is a limit, let $C=\left\{\gamma_{\nu}: \nu<\operatorname{cof} \alpha\right\}$ be increasing, closed, and cofinal in $\alpha$, with $\gamma_{0}=0$. Set $Z_{1}=\beta^{m} \times \alpha^{p} \times C$. Then $Z_{1}$ is closed in $\beta^{m} \times \alpha^{n}$, and $H\left(Z_{1}\right)$ holds because $Z_{1}$ is homeomorphic to a subspace of $\zeta^{m+1} \times \alpha^{p}$, where $\zeta=\max \{\beta$, cof $\alpha\}$. Set $Z_{2}=\left(\beta^{m} \times \alpha^{n}\right) \backslash Z_{1}$. If $\alpha=\gamma+1$, then $H\left(Z_{2}\right)$ follows from $H\left(\gamma^{m+n}\right)$. If $\alpha$ is a singular limit ordinal, then $Z_{2}=\bigoplus_{\nu<\operatorname{cof} \alpha} \beta^{m} \times \alpha^{p} \times\left(\gamma_{\nu}, \gamma_{\nu+1}\right)$. In this case, $H\left(Z_{2}\right)$ holds by induction hypothesis and Lemma 4.1 (2). Having shown $H\left(Z_{1}\right)$ and $H\left(Z_{2}\right)$, we may conclude $H\left(\beta^{m} \times \alpha^{n}\right)$ because of Lemma 4.1] 3$)$.

Lemma 4.3. Assume that $\alpha$ is regular. If $(\forall m \in \omega) H\left(\beta^{m}\right)$ for all $\beta<\alpha$, then $(\forall n \in \omega) H\left(\alpha^{n}\right)$.

Proof. We prove $(\forall m \in \omega) H\left(\beta^{m} \times \alpha^{n}\right)$ by induction on $n$. The base step $n=0$ follows from hypothesis. 
Let $n=1$. Note that for any $C$ club in $\kappa, H\left(\beta^{m} \times(\alpha \backslash C)\right)$ holds by induction hypothesis and Lemma 4.1(2). Let $H_{0}$ and $H_{1}$ be disjoint closed subsets of $X$, a normal subspace of $\beta^{m} \times \alpha$. For each $b \in \beta^{m}$, find $C_{b}$, club in $\alpha$, such that for $Y \in\left\{X, H_{0}, H_{1}\right\}$, if $Y_{b}$ is not stationary, then $C_{b} \cap Y_{p}=\emptyset$. Set $C=\bigcap\left\{C_{b}: b \in \beta^{m}\right\}$ and $X_{1}=X \cap\left(\beta^{m} \times C\right)$. Let $\pi$ be the projection: $\pi(b \frown \xi)=b$.

Set $T=\pi\left[X_{1}\right]=$ st $\pi[X]$. Lemma 3.1 implies that $T$ is normal. By $H\left(\beta^{m}\right)$, there is $W$ clopen in $T$ satisfying $\pi\left[H_{0} \cap X_{1}\right] \subseteq W \subseteq T \backslash \pi\left[H_{1} \cap X_{1}\right]$. Set $K_{0}=W \times C \cap X_{1}$ and $K_{1}=(T \backslash W) \times C \cap X_{1}$. Because $X$ is normal, there are open $U_{0}$ and $U_{1}$ such that $H_{0} \cup K_{0} \subseteq U_{0}, H_{1} \cup K_{1} \subseteq U_{1}$, and $\operatorname{cl} U_{0} \cap \operatorname{cl} U_{1}=\emptyset$. Now $X_{2}=X \backslash\left(U_{0} \cup U_{1}\right)$ is a closed, hence normal, subspace of $X$. By $H\left(\beta^{m} \times(\alpha \backslash C)\right)$, there are disjoint $W_{0}$ and $W_{1}$, clopen in $X_{2}$, satisfying

$$
\left(\operatorname{cl} U_{0} \cup H_{0}\right) \cap X_{2} \subseteq W_{0}, \quad\left(\operatorname{cl} U_{1} \cup H_{1}\right) \cap X_{2} \subseteq W_{1}, \quad \text { and } W_{0} \cup W_{1}=X_{2} .
$$

Then $U_{0} \cup W_{0}$ and $U_{1} \cup W_{1}$ are the desired clopen subsets of $X$.

Let $n=p+1$. We need

Sublemma 4.4. Let $\beta<\alpha$, let $m<\omega$, and let $C$ be club in $\alpha$. Then we have $H\left(\beta^{m} \times\left(\alpha^{n} \backslash C^{n}\right)\right)$.

Proof. Let $X$ be a normal subspace of $\beta^{m} \times\left(\alpha^{n} \backslash C^{n}\right)$. We will find a finite family of clopen, strongly zero-dimensional, subspaces which cover $X$. Set $n^{*}=$ $\{m+1, \ldots, m+n\}$. By induction hypothesis and Lemma 4.1, $H\left(Z_{i}\right)$ for all $i \in n^{*}$, where $Z_{i}=\left\{z \in \beta^{m} \times \alpha^{n}: i \notin C\right\}$.

For $x \in X$, set $\sigma(x)=\left|\left\{i \in n^{*}: x_{i} \notin C\right\}\right|$. For each nonempty $s \subseteq n^{*}$, define closed sets $H_{s}=\left\{x \in X:(\forall i \notin s)\left(x_{i} \in C\right)\right\}$ and $K_{s}=\left\{x \in X: x_{\min (s)} \in C\right\}$. Note that $H_{s} \cap K_{s} \cap\{x:|s| \leq \sigma(x)\}=\emptyset$.

We will define $X_{j}$, a clopen subspace of $X$ satisfying $\sigma(x) \geq j$ for all $x \in X_{j}$, by induction for $1 \leq j \leq n$. Set $X_{1}=X$. For each $s \in\left[n^{*}\right]^{j}$, use the normality of $X_{j}$ to find $U_{s}$, open in $X_{j}$, satisfying

$$
X_{j} \cap H_{s} \subseteq U_{s} \subseteq \operatorname{cl} U_{s} \subseteq X_{j} \backslash K_{s} .
$$

Because $\operatorname{cl} U_{s}$ is a normal subspace of $Z_{\min s}$, there is $W_{s}$, clopen in $\operatorname{cl} U_{s}$ such that $H_{s} \subseteq W_{s} \subseteq U_{s}$. Then $W_{s}$ is clopen in $X$. Set $X_{j+1}=X_{j} \backslash \bigcup\left\{W_{s}:|s|=j\right\}$. Set $W_{n^{*}}=X_{n}$. Then $X=\bigcup\left\{W_{s}: \emptyset \neq s \subseteq n^{*}\right\}$, as promised.

Returning to the proof of the case $n=p+1$, set $Z_{1}=\beta^{m} \times \operatorname{Dg}[\alpha]$. We have $H\left(Z_{1}\right)$ because $Z_{1}$ is homeomorphic to $\beta^{m} \times \alpha$. Set $Z_{2}=\beta^{m} \times \alpha^{n} \backslash Z_{1}$. Towards showing $H\left(Z_{2}\right)$, let $Y$ be a normal subspace of $Z_{2}$. For each $b \in \beta^{m}, Y_{b}$ is a normal subspace of $\alpha^{n}$ with $\Delta\left(Y_{b}\right)=\emptyset$. Apply Lemma 3.5 to get a club $C_{b}$. Set $C=\bigcap\left\{C_{b}: b \in \beta^{m}\right\}$. From Sublemma 4.4 we conclude that $Y$ is strongly zero-dimensional. Having established $H\left(Z_{2}\right)$, we apply Lemma 4.1 3 (3) to finish the proof.

\section{COllectionwise NORMAl AND SHRINKInG}

Let $J(Z)$ denote "if $X \subseteq Z$ is normal and strongly zero-dimensional, then $X$ is collectionwise normal and shrinking". To prove (2) implies (3) and (4) in Theorem 1.1, it suffices to prove $J(\Pi a)$ for all $n$-tuples $a$ of ordinals, which we do by induction on $\prec$ (see Definition 1.3). The base step is easy: if $a_{i}$ is countable for all $i \leq n$,

then every subspace of $\Pi a$ is collectionwise normal and shrinking. 
Lemma 5.1. If $J(\Pi b)$ for all $b \prec a, X$ is a normal and strongly zero-dimensional subspace of $\Pi a$, and red $_{a}(X)$, then $X$ is collectionwise normal and shrinking.

Proof. It follows directly from Lemma 3.7 .

Lemma 5.2. If $J(\Pi b)$ for all $b \prec a, X$ is a normal and strongly zero-dimensional subspace of $\Pi a$, and not red ${ }_{a}(X)$, then $X$ is collectionwise normal.

Proof. Let $\mathcal{H}$ be a discrete closed family in $X$. For each $x \in X$, choose $f(x)<x$ so that $(f(x), x]$ meets at most one $H$ in $\mathcal{H}$. Let $q$ and $R$ be as in Lemma 3.10 Because $R$ is directed and cofinal in $X$, there is at most one $H$, call it $H^{*}$, which meets $[q, a)$. Because $X$ is strongly zero-dimensional, there is a clopen $W$ such that $\bigcup\left(\mathcal{H} \backslash\left\{H^{*}\right\}\right) \subseteq W \subseteq X \backslash\left(H^{*} \cup[q, a)\right)$. For each $i$, set $Z_{i}=\left\{z \in \Pi a: z_{i}<q_{i}\right\}$. By induction hypothesis, $J\left(Z_{i}\right)$ for each $i$, which gives that $W$ is collectionwise normal. Let $\left\{U(H): H \in \mathcal{H} \backslash\left\{H^{*}\right\}\right\}$ separate $\mathcal{H} \backslash\left\{H^{*}\right\}$ in $W$. Set $U\left(H^{*}\right)=X \backslash W$. Then $\{U(H): H \in \mathcal{H}\}$ is the desired separation of $\mathcal{H}$.

Lemma 5.3. If $J(\Pi b)$ for all $b \prec a, X$ is a normal and strongly zero-dimensional subspace of $\Pi a$, and not red $_{a}(X)$, then $X$ is shrinking.

Proof. Let $\mathcal{U}$ be an open cover of $X$. For each $x \in X$, choose $U_{x} \in \mathcal{U}$ and $f(x)<x$ satisfying $(f(x), x] \subseteq U_{x}$. Let $q$ and $R$ be as in Lemma 3.10. Apply Lemma 3.9 to $[q, a) \cap X$ to obtain $Y$, a $k$-stationary set, and $\varphi$, an order preserving homeomorphism of $Y$ onto a closed, cofinal subset of $X$. For each $U \in \mathcal{U}$ set

$$
G(U)=\left\{y \in Y:(\exists x \in R)\left(\varphi(y) \leq x \text { and } U=U_{x}\right)\right\} .
$$

If $y^{\prime} \leq y \in G(U)$, then $y^{\prime} \in G(U)$; hence, $G(U)$ is open.

Sublemma 5.4. There is $\mathcal{F}=\left\{F_{U}: U \in \mathcal{U}\right\}$, a family of closed subsets of $Y$ such that $F_{U} \subseteq G(U)$ for all $U \in \mathcal{U}$ and $\bigcup \mathcal{F}=Y^{\prime}$, a final segment of $Y$.

Proof. If $Y=\{0\}$, then set $F_{U}=\{0\}$ if $\varphi(0) \in U$ and $F_{U}=\emptyset$ if $0 \notin U$. If $Y \subseteq \kappa$, set $T=\emptyset=\operatorname{st} \pi[Y]$. For each $A \subseteq \kappa$, let $A_{\emptyset}=A$. Otherwise, $Y$ is $k \frown \kappa$-stationary. Set $T=$ st $\pi[Y]$. For each $U \in \mathcal{U}$, define $U_{T}=\left\{t \in T: \sup G(U)_{t}=\kappa\right\}$, open in $T$ because $|\Pi k|<\kappa$. We split into subcases.

Subcase 1. $\bigcup\left\{U_{T}: U \in \mathcal{U}\right\}=T$. By Lemma 3.1, $T$ is normal. So by induction hypothesis, $T$ is shrinking. Let $\left\{V_{T}(U): U \in \mathcal{U}\right\}$ be a shrinking of $\left\{U_{T}: U \in \mathcal{U}\right\}$, and set $F_{U}=\left(\operatorname{cl} V_{T}(U) \times \kappa\right) \cap Y$. Then $\bigcup \mathcal{F}=Y=Y^{\prime}$.

Subcase 2. There is $q^{*} \in T \backslash \bigcup\left\{U_{T}: U \in \mathcal{U}\right\}$. Set $Y^{\prime}=\left\{y^{\prime} \in Y: q^{*} \leq \pi\left(y^{\prime}\right)\right\}$. Note that $[y, k) \backslash \bigcup\left\{G(U): U \in \mathcal{U}^{\prime}\right\} \neq \emptyset$ for all $\mathcal{U}^{\prime} \in[\mathcal{U}]^{<\kappa}$ and $y \in Y^{\prime}$. Well order $Y^{\prime}$ as $\left\{y_{\nu}: \nu<\kappa\right\}$. Inductively choose $U_{\nu} \in \mathcal{U} \backslash\left\{U_{\mu}: \mu<\nu\right\}$ so that $y_{\nu} \in G\left(U_{\nu}\right)$. Set $F_{U_{\nu}}=\left[q^{*}, y_{\nu}\right] \cap Y$; set $F_{U}=\emptyset$ if $U$ is not a $U_{\nu}$. Then $\bigcup \mathcal{F}=Y^{\prime}$.

For each $U \in \mathcal{U}$, use the normality of $X$ to find an open $V_{1}(U)$ satisfying

$$
\varphi\left[F_{U}\right] \subseteq V_{1}(U) \subseteq \operatorname{cl} V_{1}(U) \subseteq U .
$$

For each $y \in Y^{\prime}$, choose $U_{y}^{\prime} \in \mathcal{U}$ and $f^{\prime}(y) \in \Pi a^{*}$ which satisfy $\left(f^{\prime}(y), \varphi(y)\right] \cap X \subseteq$ $V_{1}\left(U_{y}^{\prime}\right)$. Apply Lemma 3.10 to $f^{\prime}$ and $Y^{\prime}$ to obtain $q^{\prime}$. Set $X_{2}=X \backslash \bigcup\left\{V_{1}(U)\right.$ : $U \in \mathcal{U}\}$. Because $\left[q^{\prime}, a\right) \cap X_{2}=\emptyset$, we have $\operatorname{red}_{a}\left(X_{2}\right)$. Hence $X_{2}$ is shrinking, and there is a closed family $\left\{F_{U}^{\prime}: U \in \mathcal{U}\right\}$ covering $X_{2}$ satisfying $F_{U}^{\prime} \subseteq U$ for all $U \in \mathcal{U}$. By normality of $X$, find open sets $V_{2}(U)$ such that

$$
F_{U}^{\prime} \subseteq V_{2}(U) \subseteq \operatorname{cl} V_{2}(U) \subseteq U
$$

for all $U \in \mathcal{U}$. Then $\left\{V_{1}(U) \cup V_{2}(U): U \in \mathcal{U}\right\}$ is the desired shrinking of $\mathcal{U}$. 
Together, the lemmas of this section yield "If $J(\Pi b)$ for all $b \prec a$, then $J(\Pi a)$ ", from which we conclude $J(\Pi a)$ for all $n$-tuples of ordinals. This completes the proof of Theorem 1.1.

Discussions with Adrienne Stanley and Nobuyuki Kemoto were helpful in preparing this article.

\section{REFERENCES}

[1] Engelking, R. General Topology, Heldermann Verlag, Berlin, 1989. MR 91c:54001

[2] Fleissner, W. Metacompact subspaces of products of ordinals, Proc. Amer. Math. Soc. 130(2002)293-301. MR 2002h:54021

[3] Fleissner, W., Kemoto, N., Terasawa, J. Strong Zero-Dimensionality in Products of Ordinals, submitted.

[4] Kemoto, N., Nogura, T., Smith K., and Yajima Y., Normal subspaces in products of two ordinals, Fund. Math. 151(1996) 279-297. MR 98b:54011

[5] Kemoto, N., Smith, K., and Szeptycki, P. Countable paracompactness versus normality in subspaces of $\omega_{1}^{2}$, Topol. Appl. 104(2000)141-154. MR 2001e:54045

[6] Kemoto, N. and Smith, K. Hereditary countable metacompactness in finite and infinite product spaces of ordinals, Topol. Appl. 77(1997)57-63. MR 98j:54041

[7] Kunen, K. Set Theory, An Introduction to Independence Proofs, Elsevier, 1980. MR 82f:03001

[8] Stanley, A., Normal subspaces of finite products of ordinals, Abstracts AMS 19(1998)474.

Department of Mathematics, University of Kansas, Lawrence, Kansas 66045

E-mail address: fleissne@math.ukans.edu 